\title{
Negative Permittivity Behavior in Flexible Carbon Nanofibers- Polydimethylsiloxane Films
}

Haikun Wu, ${ }^{1,2}$ Haowei Sun, ${ }^{1}$ Fengjin Han, ${ }^{1}$ Peitao Xie,,${ }^{1, *}$ Yiming Zhong, ${ }^{1}$ Bin Quan, ${ }^{3}$ Yaman Zhao, ${ }^{4}$ Chunzhao Liu,,${ }^{1,}$ Runhua Fan ${ }^{4}$ and Zhanhu $\mathrm{Guo}^{5}$

\begin{abstract}
Flexible electronic devices have recently become a research hotspot due to their potential in different applications. However, there is a lack of studies about flexible metamaterials with negative parameters. In this work, the composite film with carbon nanofibers (CNFs) dispersed in a polydimethylsiloxane (PDMS) matrix is designed to construct flexible metamaterials with negative permittivity, showing excellent mechanical durability and flexibility. The microstructures, mechanical durability, alternating current conductivity $\left(\sigma_{\mathrm{ac}}\right)$, and permittivity $\left(\varepsilon^{\prime}\right.$ and $\left.\varepsilon^{\prime \prime}\right)$ were investigated and discussed in detail. A transition of conduction mechanism from jumping conduction to metal-like conduction was observed when the CNFs content was improved from 6 to $14 \mathrm{wt} \%$. The CNFs-PDMS film with CNFs content of $14 \mathrm{wt} \%$ achieved negative permittivity over the whole frequency, which resulted from the construction of a conductive CNFs network. The equivalent circuit models were used to analyze the impedance $\left(Z^{\prime}\right.$ and $\left.Z^{\prime \prime}\right)$ behavior of the composites to determine the mechanism of negative permittivity. This study will provide theoretical and technical support for the design of flexible metamaterials and promote their practical applications in wearable electronic devices.
\end{abstract}

Keywords: Negative permittivity; Silicone rubber; Carbon nanofibers; Flexible film.

Received: 29 August 2021; Revised: 18 November 2021; Accepted: 20 November 2021.

Article type: Research article.

\section{Introduction}

In the past few years, silicone rubber (SR)-based composites have received much attention due to their unique characteristics, such as low toxicity, chemical stability, lowtemperature toughness, and low-surface energy. ${ }^{[1-5]}$ As for constructing SR-based composites, the nature, properties, and dispersion of the filler, and interfacial interaction between filler and SR matrix are all significant for determining the final performance of composites..$^{[6-10]}$ For example, metal nanoparticles, nanoscale carbon materials, and silicates are incorporated into the SR matrix to fabricate SR-based composites, showing enhanced mechanical, electrical, thermal, and other functional properties. ${ }^{[11-15]}$ Due to these excellent

\footnotetext{
${ }^{1}$ State Key Laboratory of Bio-fbers and Eco-textiles Institute of Biochemical Engineering, College of Materials Science and Engineering, Qingdao University, Qingdao 266071, China.

2 Hong Kong Branch of National Precious Metals Material Engineering Research Centre (NPMM), Department of Chemistry, City University of Hong Kong, Kowloon, Hong Kong, China. ${ }^{3}$ Institute of Advanced Materials and Flexible Electronics (IAMFE), School of Chemistry and Materials Science, Nanjing University of Information Science \& Technology Nanjing 210044, China.
}

performances, SR-based composites exhibit great potential in applications of artificial skin, aerospace, electromagnetic interference shielding and absorbing, etc. ${ }^{[16-18]}$

Among carbon materials, carbon nanofibers (CNFs) exhibit excellent mechanical strength and electrical properties, which are attributed to CNFs' graphite ordering and dimension. ${ }^{[19]}$ Besides, the properties of CNFs are related to their diameters and lengths, which are in the order of micrometers. As a result, CNFs are usually used to prepare SR-based composites with a lot of applications. Roy et al. ${ }^{[20]}$ have reported that the tensile strength and modulus of carbon nanofibers-polydimethylsiloxane (CNFs-PDMS) composites are improved by $150 \%$ and $310 \%$ respectively, compared to pure PDMS. Enhanced electrical conductivity (in the order of $10^{-8} \mathrm{~S} \cdot \mathrm{cm}^{-1}$ ) of CNFs-PDMS composites is reported, which is assigned to the better dispersion of CNFs. ${ }^{[21]}$

However, CNFs used as fillers in the SR matrix to change dielectric properties and achieve negative permittivity are rarely investigated and reported. Due to intrinsic properties such as lower loss, outstanding electricity, excellent transparency, and good mechanical property, PDMS is regarded as a promising candidate to construct flexible and wearable devices. In this paper, the CNFs-PDMS films with 
excellent mechanical durability and flexibility were prepared to achieve negative permittivity and exhibit great potential in the fields of electromagnetic functional materials and wearable electronics. The microstructures of CNFs and CNFsPDMS films were characterized by field emission scanning electron microscopy (FESEM). Mechanical durability and flexibility were tested under various mechanical deformations. The dielectric properties including the conduction mechanism and negative permittivity behavior were investigated. The equivalent circuit models from impedance $\left(Z^{\prime}\right.$ and $\left.Z^{\prime \prime}\right)$ were used to analyze the mechanism of the negative permittivity.

\section{Experimental}

\subsection{Procedures}

The in-situ polymerization process was used to fabricate carbon nanofibers (CNFs)-polydimethylsiloxane (PDMS) films. The silicone (Sylgard 184 A, Dow Corning Company), n-heptane, and curing agent (Sylgard 184B, Dow Corning Company) were mixed together with a 10:10:1 weight ratio in a beaker. Different contents of CNFs $(6,8,10,12$, and $14 \mathrm{wt} \%)$ were incorporated into the mixed solution and ultrasonic treatment at room temperature was performed to allow CNFs to disperse uniformly in the solution. After that, the film applicator (Elcometer 3530) was used to coat the slurry on the glass substrate. The film with a thickness of $100 \mu \mathrm{m}$ was dried at $353 \mathrm{~K}$ for 2 hours in the oven. And then the CNFs-PDMS film was taken out and peeled off from the substrate.

\subsection{Characterization}

The microstructures of CNFs-PDMS films were observed by the FESEM (Hitachi, SU-70, Tokyo, Japan). The alternating current conductivity $\left(\sigma_{\mathrm{ac}}\right)$, complex permittivity $\left(\varepsilon^{\prime}, \varepsilon^{\prime \prime}\right)$, and the impedance $\left(Z^{\prime}, Z^{\prime \prime}\right)$ of the CNFs-PDMS films were measured by the impedance analyzer (Agilent E4991A). The real part of permittivity $\left(\varepsilon^{\prime}\right)$ and imaginary part of permittivity ( $\left.\varepsilon^{\prime \prime}\right)$ were obtained from the formula $\varepsilon^{\prime}=C d / A \varepsilon_{0}$ and $\varepsilon^{\prime \prime}=$ $d / 2 \pi f A \varepsilon_{0}$, where $C$ is the output data, representing the capacitance, $d$ is the thickness of CNFs-PDMS film, $A$ is the area of the electrode, $\varepsilon_{0}$ is the permittivity of vacuum $\left(8.85 \times 10^{-12} \mathrm{~F} / \mathrm{m}\right), f$ is the tested frequency. Alternating current conductivity was obtained by $\sigma_{\text {ac }}=d / R A$, where $R$ is output data, representing the resistance. The real part of the impedance

$\left(Z^{\prime}\right)$ and imaginary parts of impedance $\left(Z^{\prime \prime}\right)$ were measured directly.

\footnotetext{
${ }^{4}$ College of Ocean Science and Engineering, Shanghai Maritime

University, Shanghai.

${ }^{5}$ Integrated Composites Laboratory (ICL), Department of Chemical and Biomolecular Engineering, University of Tennessee, Knoxville, Tennessee 37996, United States.

*E-mail: xiepeitao1991@qdu.edu.cn (P.Xie); czliu@qdu.edu.cn (C. Liu)
}

\section{Results and discussion}

\subsection{Microstructure of the CNFs-PDMS films}

The microstructures of CNFs-PDMS films with different CNFs contents are shown in Fig. 1. From Fig. 1a, it was observed that CNFs exhibited cylindrical nanostructures with diameter in the order of nanometers and lengths in the order of micrometers, the CNFs stacked different arrangements in the form of herringbone or ribbon. When CNFs were incorporated into the PDMS matrix to form CNFs-PDMS film with CNFs content of $6 \mathrm{wt} \%$ (Fig. 1b), CNFs were observed to be dispersed uniformly in the PDMS matrix and isolated by the insulating matrix. The CNFs with good electrical conductivity and insulating PDMS matrix could be equivalent to microcapacitors, so there were many equivalent micro-capacitors in CNFs-PDMS films. When CNFs content was increased, more CNFs were distributed in the insulating matrix, indicating that more equivalent micro-capacitors were formed in CNFsPDMS films. As shown in Fig. 1f, when CNFs content was improved to $14 \mathrm{wt} \%$, CNFs connected to each other to form continuous pathways in the film. These results indicated that microstructures, equivalent micro-capacitors, and conductive pathways in CNFs-PDMS films could be adjusted by changing CNFs content.

Photographs of the CNFs-PDMS film under various mechanical deformations such as cutting, winding, multitwisting, folding and multi-folding are shown in Fig. 2. From Fig. 2a, it was observed that the CNFs-PDMS film was easy to cut, just like paper, exhibiting good processibility, hence, the composite film can be processed into different shape when used as flexible electronic devices. As shown in Fig. $2 b$ and Fig. 2c, the CNFs-PDMS film under winding and multitwisting still exhibited good mechanical stability and durability. Even under folding and multi-folding (Fig. 2d and e), the CNFs-PDMS film was still not broken down, indicating excellent flexibility and robustness. The excellent mechanical durability and high flexibility allowed the CNFs-PDMS film to exhibit applications for wearable electronic devices.

\subsection{Alternating current conductivity of the CNFs-PDMS films}

Figure 3 exhibits the frequency dependence of alternating current conductivity $\left(\sigma_{\mathrm{ac}}\right)$ for CNFs-PDMS films with different CNFs contents. It was observed that $\sigma_{\text {ac }}$ went up as the CNFs content increased from 6 to $14 \mathrm{wt} \%$ within the tested frequency range, which was attributed to the addition of CNFs with good electrical conductivity. For CNFs-PDMS films with CNFs content from 6 to $12 \mathrm{wt} \%$, the $\sigma_{\text {ac }}$ increased with frequency, following the Jonscher power law: $\sigma(\omega) \propto A \omega^{n},{ }^{222-}$ ${ }^{24]}$ where $A$ is the pre-exponential factor, $\omega(\omega=2 \pi f)$ represents the angular frequency, and $n$ is the fractional exponent. The fitted results using the formula were shown as solid red lines in Fig. 3, and the R-square of fitted results for CNFs-PDMS films with CNFs contents from 6 to $12 \mathrm{wt} \%$ was $0.99,0.98$, 0.98 , and 0.96 respectively, indicating that the experimental data were strongly relevant to Jonscher power law and thus 

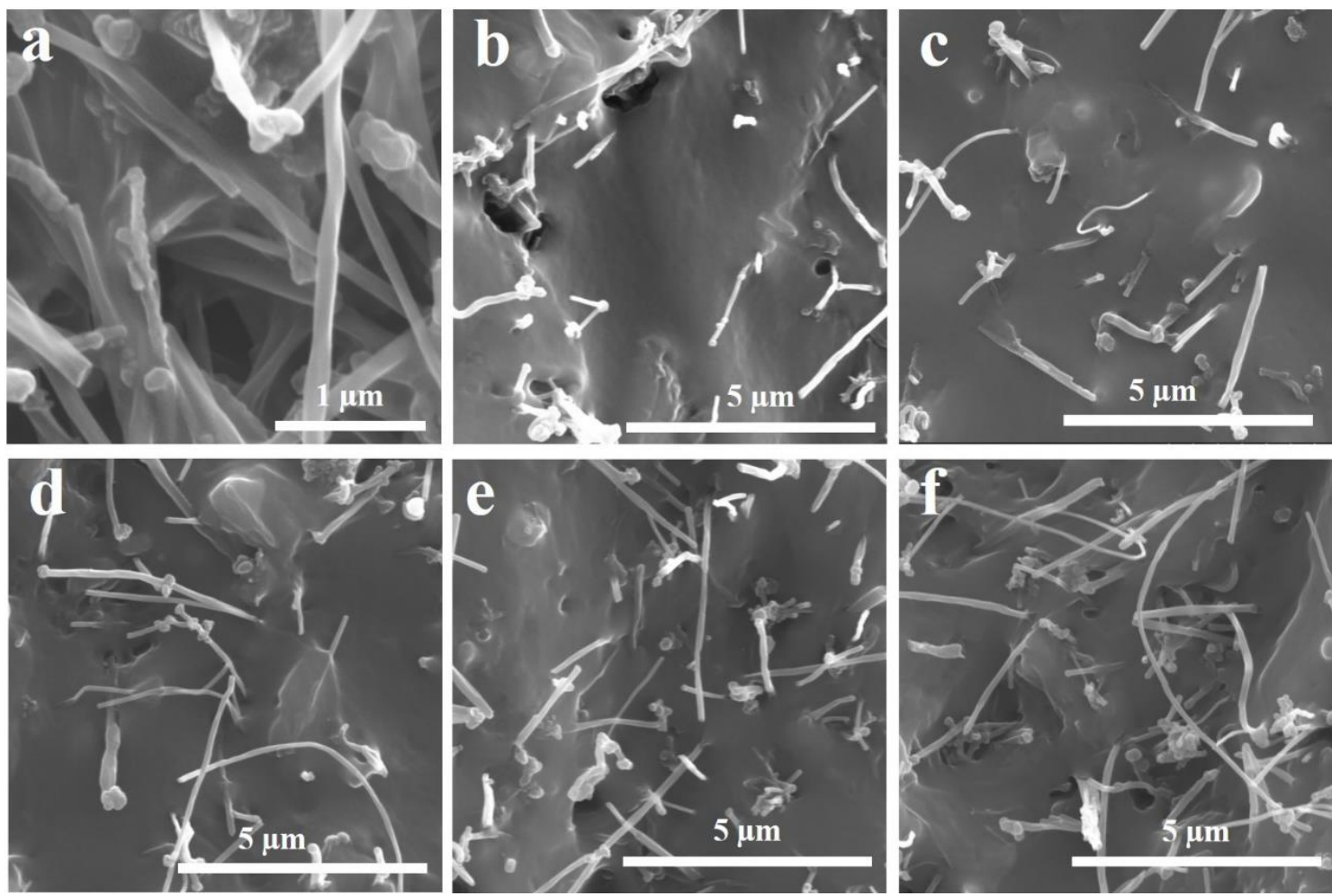

Fig. 1 FESEM images of the carbon nanofibers (CNFs) powders (a) and carbon nanofibers-polydimethylsiloxane (CNFs-PDMS) films with different CNFs contents of $6 \mathrm{wt} \%$ (b), $8 \mathrm{wt} \%$ (c), $10 \mathrm{wt} \%$ (d), $12 \mathrm{wt} \%$ (e), and $14 \mathrm{wt} \%$ (f).

hopping conduction behavior played a significant role in these composite films. ${ }^{[25-27]}$ These results were consistent with the results of microstructures, because CNFs were isolated by an insulating matrix, and electrons could just jump between isolated CNFs for films with CNFs content from 6 to $12 \mathrm{wt} \%$. However, when CNFs content was increased to $14 \mathrm{wt} \%$, the $\sigma_{\mathrm{ac}}$ exhibited a direct-current (dc) conductivity plateau and decreased with frequency in the high-frequency range. This indicated another conduction mechanism: metal-like conduction behavior, attributed to the formation of conductive CNFs network in the composite films. ${ }^{[28]}$
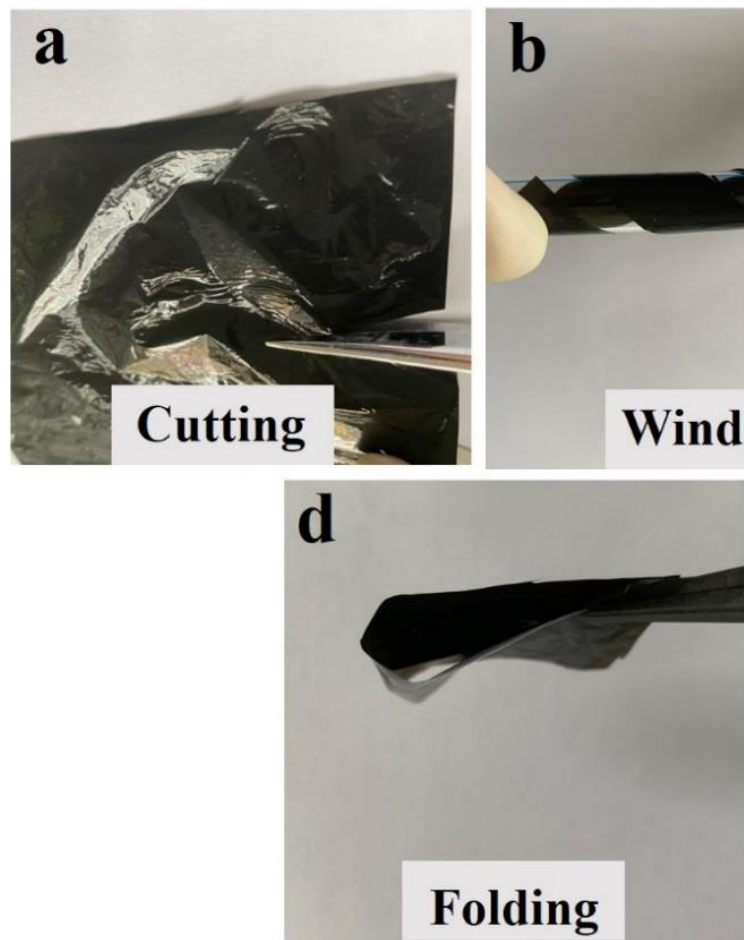
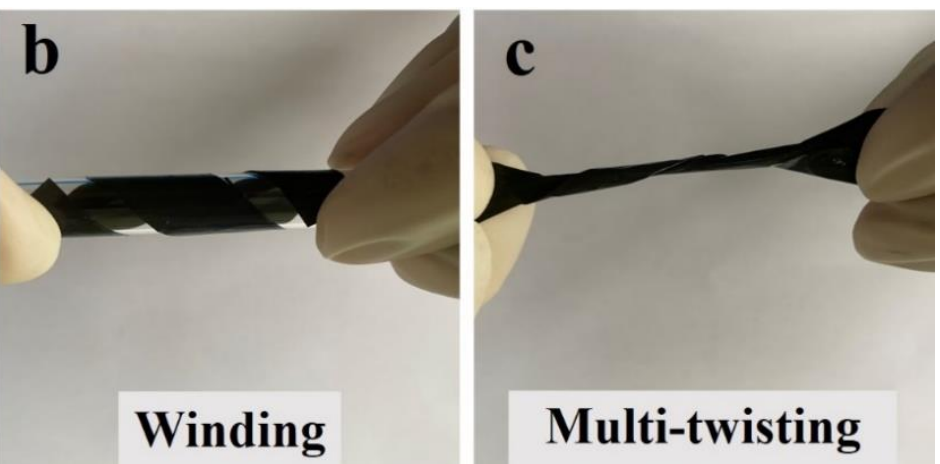

Fig. 2 The photographs of highly flexible CNFs-PDMS films with different operations. (a) Cutting, (b) Winding, (c) Multi-twisting, (d) Folding, and (e) Multi-folding. 


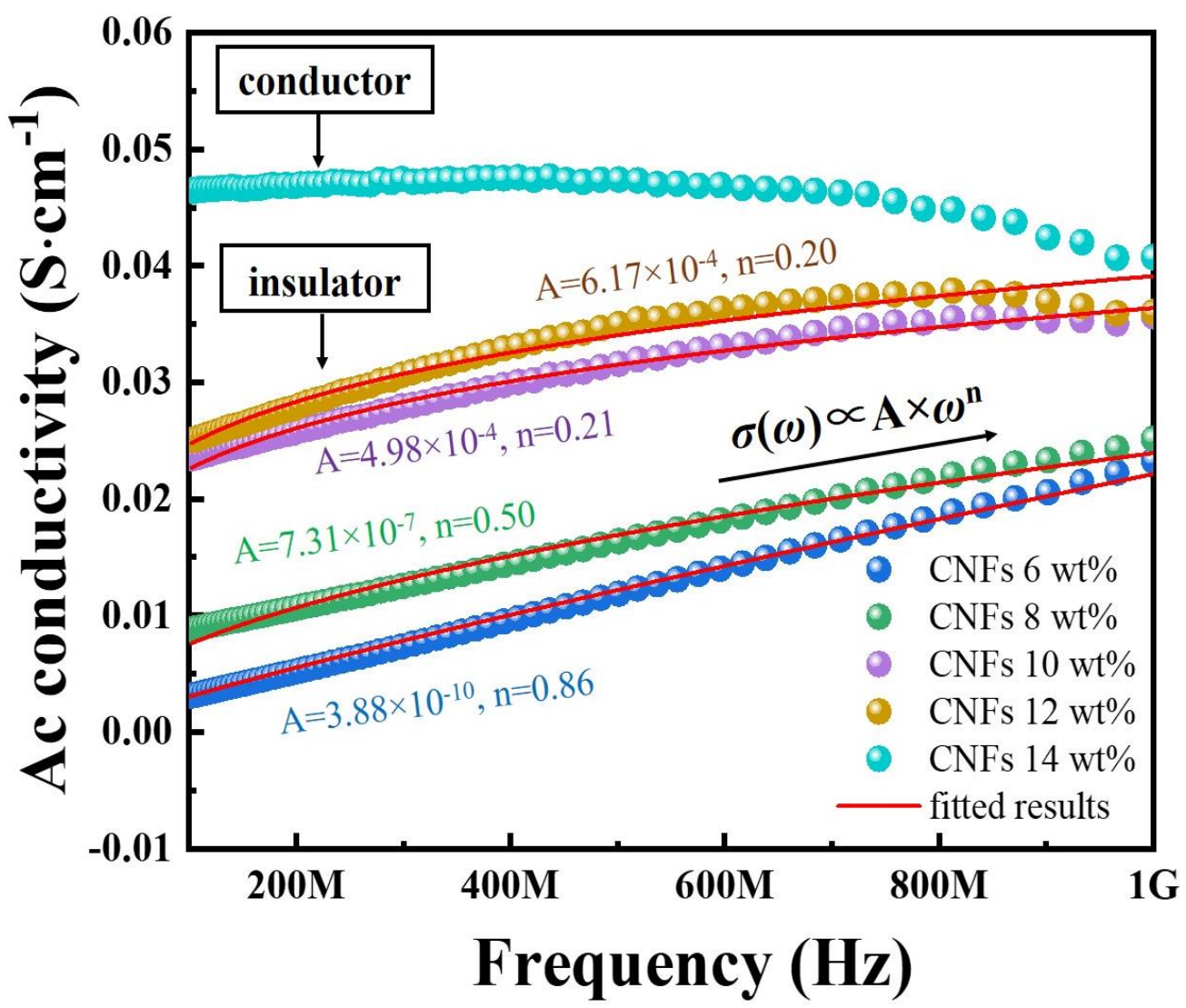

Fig. 3 Frequency dependences of alternating current conductivity $\left(\sigma_{\mathrm{ac}}\right)$ from the CNFs-PDMS films with different CNFs contents of $6,8,10,12$, and $14 \mathrm{wt} \%$.

\subsection{Dielectric properties of the CNFs-PDMS films}

The frequency dispersions of real permittivity $\left(\varepsilon^{\prime}\right)$ for the CNFs-PDMS films with different CNFs contents are shown in Fig. 4a. For films with CNFs content from 6 to $12 \mathrm{wt} \%$, it was found that the value of $\varepsilon^{\prime}$ was positive over the whole frequency, and the $\varepsilon^{\prime}$ increased with increasing CNFs content at low frequencies. When CNFs content was low, the CNFs isolated by insulating PDMS matrix could be equivalent to micro-capacitor electrodes, which was observed and discussed from microstructure results. Moreover, interfacial polarization appeared between CNFs and PDMS due to the accumulation of charge carriers in the interface under the external electrical field. ${ }^{[29,30]}$ The micro-capacitors effect and interfacial polarization effect were enhanced with the increase of CNFs content, leading to the improvement of positive permittivity. In dielectric materials, the macroscopic electric field $\left(E_{\mathrm{M}}\right)$ is composed of two parts, that is, tested electrical field $\left(E_{\mathrm{T}}\right)$ and electrical field $\left(E_{\mathrm{D}}\right)$ caused by surface-bound charges, $E_{\mathrm{M}}=E_{\mathrm{T}}$ $+E_{\mathrm{D}}$. The direction of $E_{\mathrm{D}}$ is opposite to that of $E_{\mathrm{T}}$ in the materials with positive permittivity, so $E_{\mathrm{M}}<E_{\mathrm{T}}$ and $E_{\mathrm{D}}$ are also called depolarization fields. ${ }^{[31]}$ From Fig. 4a, it was also found that the $\varepsilon^{\prime}$ decreased with the frequency, which was attributed to the dielectric relaxation behavior at a higher frequency. ${ }^{[32,33]}$

However, when CNFs content was increased to $14 \mathrm{wt} \%$, it was observed that the $\varepsilon^{\prime}$ was negative within the tested frequency, resulting from the formation of a conductive CNFs network in the film. In the materials with negative permittivity, the direction of $E_{\mathrm{D}}$ is the same as that of $E_{\mathrm{T}}$, so $E_{\mathrm{M}}>E_{\mathrm{T}}$, and this phenomenon is also called the Local electric field enhancement effect. ${ }^{[31]}$ The type of negative permittivity was not consistent well with the Drude model which was usually used to describe the negative permittivity behavior in the metal conductive network. ${ }^{[34,35]}$ It was observed that the negative permittivity showed a small negative value $\left(0<\varepsilon^{\prime}<-30\right)$, moreover, the negative permittivity value was less dependent on frequency compared with the typical Drude-type ones, which exhibited great potentials in double negative metamaterials, electromagnetic shielding and none-winding inductor due to good impedance matching behavior. The negative permittivity could be explained by the combination of Lorentz-type dielectric behavior and Drude-type behavior. The dielectric constant was given: ${ }^{[36,37]}$

$$
\begin{aligned}
\varepsilon_{\mathrm{r}}^{\prime}=1-\omega_{\mathrm{p}}^{2} /\left(\omega^{2}+\Gamma_{\mathrm{D}}^{2}\right) & +K \omega_{\mathrm{L}}^{2}\left(\omega_{\mathrm{L}}^{2}-\omega^{2}\right) /\left(\left(\omega_{\mathrm{L}}^{2}-\omega^{2}\right)^{2}\right. \\
& \left.+\Gamma_{\mathrm{L}}^{2} \omega^{2}\right)
\end{aligned}
$$

where, $\Gamma_{\mathrm{D}}$ is the damping constant, $\omega_{\mathrm{p}}=2 \pi f_{\mathrm{p}}$ is the plasmon angular frequency, $\Gamma_{\mathrm{L}}$ is the damping constant of Lorentz resonance, $\omega_{\mathrm{L}}=2 \pi f_{\mathrm{L}}$ is the Lorentz resonance angular frequency, and $K$ is the dc electric susceptibility. Different dispersion of CNFs resulted in different dielectric responses, conductive CNFs networks made contributions to Drude-type dielectric response while isolated CNFs made more contributions to Lorentz-type dielectric response. ${ }^{[37]}$ Therefore, these results showed that the appearance of negative permittivity was affected directly by microstructures. 

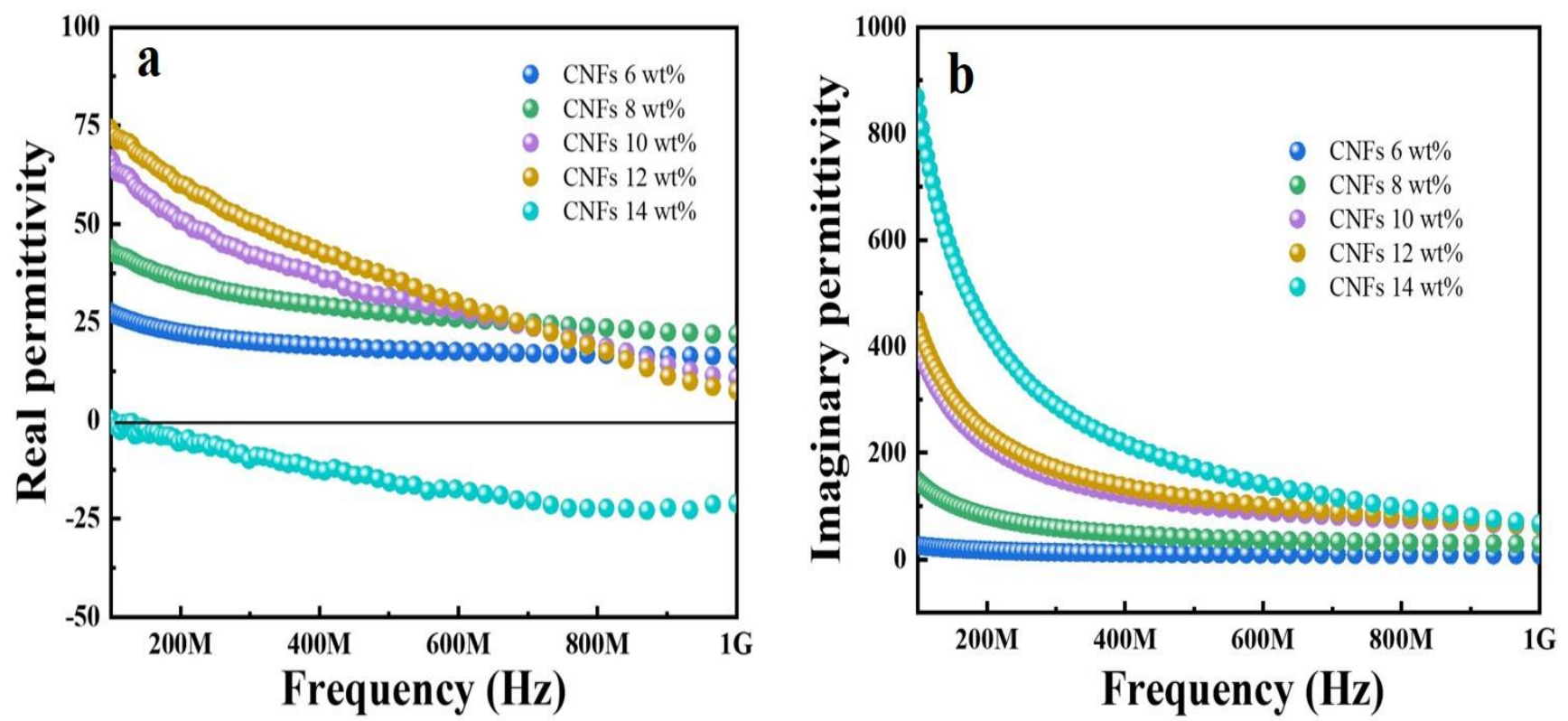

Fig. 4 (a) Frequency dependences of the real permittivity $\left(\varepsilon^{\prime}\right)$ for the CNFs-PDMS films with different CNFs contents from 6 to 14 $\mathrm{wt} \%$. (b) Frequency dependences of the imaginary permittivity $(\varepsilon ")$ from the CNFs-PDMS films with different CNFs contents from 6 to $14 \mathrm{wt} \%$.

The frequency dispersions of imaginary permittivity $\left(\varepsilon^{\prime \prime}\right)$ for the CNFs-PDMS films with different CNFs contents are shown in Fig. 4b. The $\varepsilon^{\prime \prime}$ increased with increasing CNFs contents from 6 to $14 \mathrm{wt} \%$, which was ascribed to the enhancement of dielectric loss in films. The dielectric loss mainly resulted from interfacial polarization loss and conduction loss. ${ }^{[38-44]}$ For films with CNFs content from 6 to $12 \mathrm{wt} \%$, the interfacial polarization effect was obvious and enhanced with increasing CNFs content, so the $\varepsilon^{\prime \prime}$ increased and interfacial polarization loss was the main dielectric loss mechanism in these films. However, for the film with CNFs content of $14 \mathrm{wt} \%$, the continuous conductive network was formed and the conduction current was generated, resulting in the conduction loss which mainly contributed to the dielectric loss mechanism. The flexible CNFs-PDMS film with negative permittivity and relatively low dielectric loss could be a promising candidate to make wearable sensors, new antennae, perfect lenses, and electromagnetic interference shielding materials. ${ }^{[45-50]}$

\subsection{Impedance and equivalent circuit analysis of the CNFs- PDMS films}

The equivalent circuit analysis based on impedance spectra of CNFs-PDMS films was performed to study the influence of microstructures on negative permittivity behavior and mechanisms (Fig. 5). From Fig. 5a to Fig. 5d, it was found that the imaginary part of impedance, which represents the reactance, was negative over the whole frequency for films with CNFs content from 6 to $12 \mathrm{wt} \%$, exhibiting a capacitive behavior. The fitted results, which were based on their equivalent circuit models consisting of a series resistor $\left(R_{1}\right)$, a capacitor $(C)$, and a parallel resistor $\left(R_{2}\right)$, are shown as solid lines. The Chi-squared value was used to describe the correlation between simulated equivalent circuit models and experimental data, and the smaller value of the Chi-squared, the stronger correlation between simulated equivalent circuit models and experimental data. The Chi-squared statistic for films with CNFs content of $6,8,10$, and $12 \mathrm{wt} \%$ was $1.07 \times$ $10^{-2}, 2.33 \times 10^{-3}, 5.66 \times 10^{-4}$ and $1.22 \times 10^{-3}$, respectively. The capacitor $C$ in the model originated from equivalent microcapacitors in microstructures and distributed capacitance in the CNFs microregions. When CNFs content increased from 6 to $12 \mathrm{wt} \%$, it was found that the simulated $C$ value improved from $3.51 \times 10^{-12} \mathrm{~F}$ to $1.15 \times 10^{-11} \mathrm{~F}$, indicating that more equivalent micro-capacitors were formed. These results were consistent with the analysis of microstructures and dielectric constant.

When CNFs content increased to $14 \mathrm{wt} \%$ (Fig. 5e), the imaginary part of impedance became positive within the tested frequency, corresponding to inductive characteristics. Its equivalent circuit model consisted of a capacitor $C$, inductors ( $L_{1}$ and $L_{2}$ ), and resistors $\left(R_{1}\right.$ and $\left.R_{2}\right)$, the Chi-squared value was $9.07 \times 10^{-4}$. It was found that the simulated $C$ value decreased from $1.15 \times 10^{-11} \mathrm{~F}$ to $9.26 \times 10^{-12} \mathrm{~F}$ when CNFs content was increased from 12 to $14 \mathrm{wt} \%$, which was attributed to the formation of a continuous conductive CNFs network. In the process, CNFs microregions were mainly used to form conductive pathways instead of the micro-capacitor network, so the equivalent micro-capacitor effect has degenerated gradually. Inductors in the equivalent circuit model originated from conductive CNFs network and represented the conductive loop in films. The results showed that microstructures had a significant effect on dielectric properties and the negative permittivity behavior was closely related to the conductive CNFs network. 

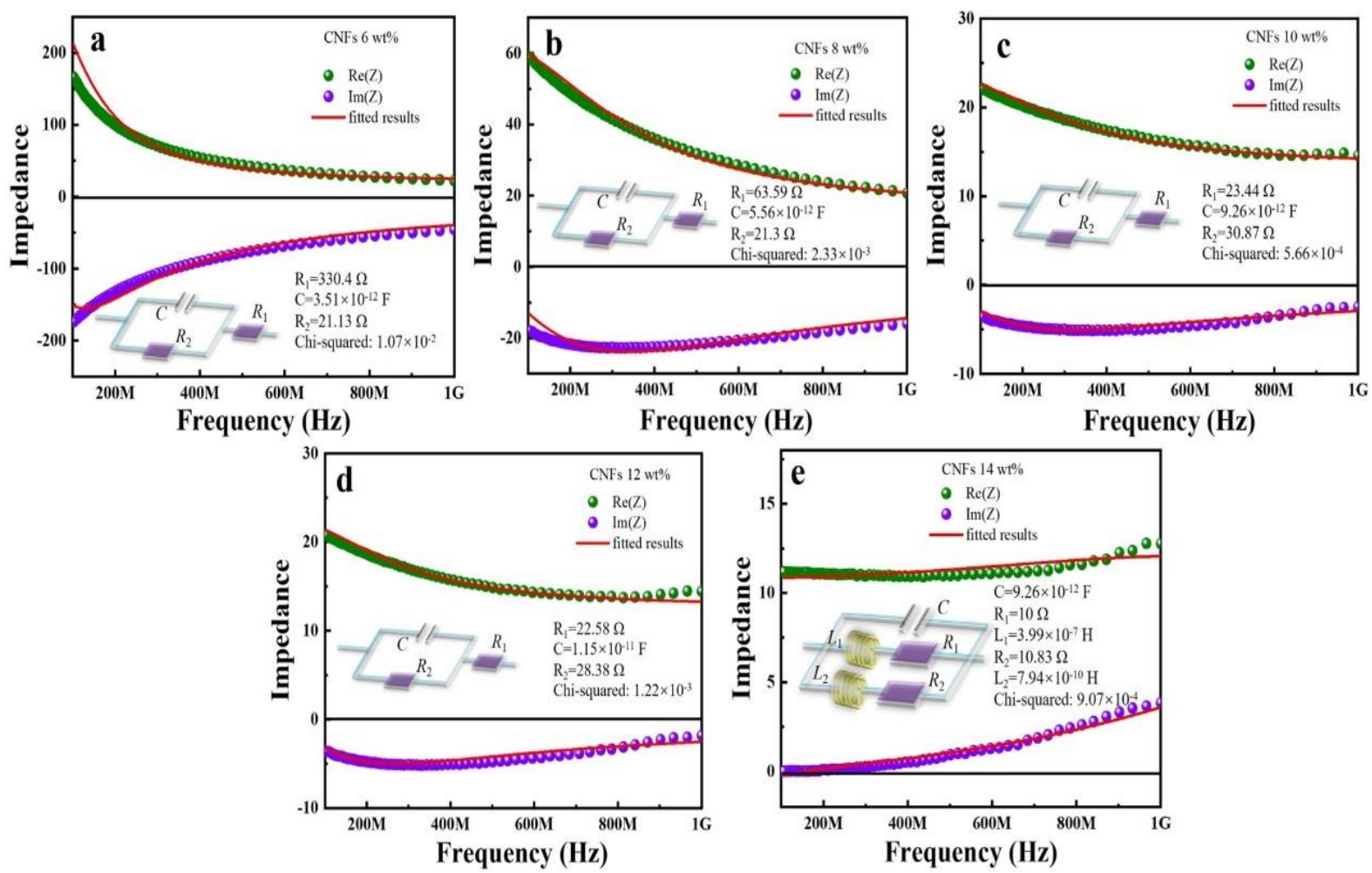

Fig. 5 Equivalent circuit analysis of the CNFs-PDMS films. CNFs-PDMS films with CNFs content from 6 to $12 \mathrm{wt} \%$ were equivalent to the circuit model consisting of a capacitor and resistors ( $a, b, c$ and d). The CNFs-PDMS film with CNFs content of $14 \mathrm{wt} \%$ was equivalent to the circuit model consisting of a capacitor, resistors, and inductors (e). Re (Z) was the real part of the impedance. $\operatorname{Im}$ (Z) was the imaginary part of the impedance.

\section{Conclusions}

In summary, the flexible CNFs-PDMS films with tunable negative permittivity and excellent mechanical durability were prepared. A continuous conductive CNFs network was observed when CNFs content was $14 \mathrm{wt} \%$ in the composite film, and the micro-capacitor effect was obvious when CNFs were below $14 \mathrm{wt} \%$. When the content of CNFs increased from $6 \%$ to $14 \mathrm{wt} \%$, a transition in the conduction mechanism from jump conduction to metal-like conduction was observed. Negative permittivity behavior was found in the CNFs-PDMS film with CNFs content of $14 \mathrm{wt} \%$ due to the formation of a conductive network, which was explained by the combination of the Lorentz model and the Drude model. The equivalent circuit model showed that inductors represented the appearance of negative permittivity. The flexible CNFsPDMS films with adjustable negative permittivity will have great significance in designing novel electromagnetic devices and intelligent materials, where excellent mechanical durability is essential for designing wearable electronic devices.

\section{Acknowledgments}

This work received financial support from the Natural Science Foundation of Shandong Province [ZR2020QE006], the Postdoctoral Applied Research Project of Qingdao, the Postdoctoral Innovation Project of Shandong Province [202003031], the China Postdoctoral Science Foundation
[2020M671992], Key Research and Development Project of Shandong Province [grant No. 2019GSF109079], National Natural Science Foundation of China [grant No. 52101176], and support from State Key Laboratory of Bio-fibers and Ecotextiles, Institute of Biochemical Engineering.

\section{Conflict of interest}

There are no conflicts to declare.

\section{Supporting information}

Not applicable.

\section{References}

[1] E. P. Giannelis, Polymer layered silicate nanocomposites, Advanced Materials, 1996, 8, 29-35, doi: 10.1002/adma.19960080104.

[2] X. Hu, H. Wu, S. Liu, S. Gong, Y. Du, X. Li, X. Lu, J. Qu, Fabrication of organic shape-stabilized phase change material and its energy storage applications, Engineered Science, 2021, 17, 1-27, doi: 10.30919/es8d474.

[3] J. Liu, J. Zhang, J. Tang, L. Pu, Y. Xue, M. Lu, L. Xu, Z. Guo, Polydimethylsiloxane resin nanocomposite coating with alternating multilayer structure for corrosion protection performance, ES Materials \& Manufacturing, 2020, 10, 29-38, doi: 10.30919/esmm5f912.

[4] T. Song, B. Jiang, Y. Li, Z. Ji, H. Zhou, D. Jiang, I. Seok, V. Murugadoss, N. Wen, H. Colorado, Self-healing materials: a 
review of recent developments, ES Materials \& Manufacturing, 2021, 14, 1-19, doi: 10.30919/esmm5f465.

[5] Y.-F. Su, G. Han, Z. Kong, T. Nantung, N. Lu, Embeddable piezoelectric sensors for strength gain monitoring of cementitious materials: the influence of coating materials, Engineered Science, 2020, 11, 66-75, doi: 10.30919/es8d1114.

[6] G. A. Ozin, Nanochemistry: Synthesis in diminishing dimensions, Advanced Materials, 1992, 4, 612-649, doi: 10.1002/adma.19920041003.

[7] S. Sharma, S. Komarneni, Synthesis and characterization of synthetic mica-bionanocomposites, Applied Clay Science, 2009 , 42, 553-558, doi: 10.1016/j.clay.2008.03.006.

[8] L. Hsu, C. Weder, S. J. Rowan, Stimuli-responsive, mechanically-adaptive polymer nanocomposites, Journal of Materials Chemistry, 2011, 21, 2812-2822, doi: 10.1039/c0jm02383c.

[9] F. Leroux, J. P. Besse, Polymer interleaved layered double hydroxide: a new emerging class of nanocomposites, Chemistry of Materials, 2001, 13, 3507-3515, doi: 10.1021/cm0110268.

[10] M. Alexandre, P. Dubois, Recent advances in incisional hernia treatment, Materials Science and Engineering: R: Reports, 2000, 28, 1-63, doi: 10.1016/S0927-796X(00)00012-7.

[11] W. Chen, B. Qu, Structural characteristics and thermal properties of PE-g-MA/MgAl-LDH exfoliation nanocomposites synthesized by solution intercalation, Chemistry of Materials, 2003, 15, 3208-3213, doi: 10.1021/cm030044h.

[12] W. Chen, L. Feng, B. Qu, in situ synthesis of poly(methyl methacrylate) $/ \mathrm{MgAl}$ layered double hydroxide nanocomposite with high transparency and enhanced thermal properties, Solid State Communications, 2004, 130, 259-263, doi: 10.1016/j.ssc.2004.01.031.

[13] H. Acharya, S. K. Srivastava, A. K. Bhowmick, Synthesis of partially exfoliated EPDM/LDH nanocomposites by solution intercalation: structural characterization and properties, Composites Science and Technology, 2007, 67, 2807-2816, doi: 10.1016/j.compscitech.2007.01.030.

[14] S. K. Srivastava, M. Pramanik, H. Acharya, Ethylene/vinyl acetate copolymer/clay nanocomposites, Journal of Polymer Science Part B: Polymer Physics, 2006, 44, 471-480, doi: 10.1002/polb.20702.

[15] M. Maiti, A. Bhowmick, New fluoroelastomer nanocomposites from synthetic montmorillonite, Composites Science and Technology, 2008, 68, 1-9, doi: 10.1016/j.compscitech.2007.05.042.

[16] T. Kuila, S. Srivastava, A. Bhowmick, Thermoplastic polyolefin based polymer-blend-layered double hydroxide nanocomposites, Composites Science and Technology, 2008, 68, 3234-3239, doi: 10.1016/j.compscitech.2008.08.003.

[17] M. Alexandru, M. Cazacu, A. Nistor, Polydimethylsiloxane/silica/titania composites prepared by solvent-free sol-gel technique, Journal of Sol-Gel Science and Technology, 2010, 56, 310-319, doi: 10.1007/s10971-010-23075.

[18] J. E. Mark, Some unusual elastomers and experiments on rubberlike elasticity, Progress in Polymer Science, 2003, 28,
1205-1221, doi: 10.1016/s0079-6700(03)00024-8.

[19] P. Yang, H. Zhao, Y. Yang, P. Zhao, X. Zhao, L. Yang, Fabrication of $\mathrm{N}$, P-codoped $\mathrm{Mo}_{2} \mathrm{C} /$ carbon nanofibers via electrospinning as electrocatalyst for hydrogen evolution reaction, ES Materials \& Manufacturing, 2020, 7, 34-39, doi: 10.30919/esmm5f618.

[20] N. Roy, A. Bhowmick, Tailor-Made Fibrous Nanohydroxyapatite/Polydimethylsiloxane Composites: Excavating the Role of Nanofiller Aspect Ratio, Amorphicity, and Noncovalent Surface Interaction, The Journal of Physical Chemistry C, 2012, 116, 8763-8772, doi: 10.1021/jp210835a.

[21] N. Roy, A. Bhowmick, In situ preparation, morphology and electrical properties of carbon nanofiber/polydimethylsiloxane nanocomposites, Journal of Materials Science, 2012, 47, 272-281, doi: 10.1007/s10853-011-5795-y.

[22] A. B. Kakade, S. K. Deshpande, S. B. Kulkarni, Electrical conductivity and modulus studies of $x[$ CNFO]-(1-x)[0.5BCT$0.5 \mathrm{BZT}]$ multiferroic with dielectric, magnetic and magnetodielectric properties, Engineered Science, 2021 18, 168-176, doi: $10.30919 / \mathrm{es} 8 \mathrm{~d} 485$.

[23] P. Xie, W. Sun, Y. Liu, A. Du, Z. Zhang, G. Wu, R. Fan, Carbon aerogels towards new candidates for double negative metamaterials of low density, Carbon, 2018, 129, 598-606, doi: 10.1016/j.carbon.2017.12.009.

[24] P. Xie, W. Sun, A. Du, Q. Hou, G. Wu, R. Fan, Epsilonnegative carbon aerogels with state transition from dielectric to degenerate semiconductor, Advanced Electronic Materials, 2021, 7, 2000877, doi: 10.1002/aelm.202000877.

[25] R. Ahmed, S. Wang, S. Rehman, J. Sun, J. Wang, R. Si, A. Zhu, Y. Yu, Q. Li, C. Wang, Maxwell-Wagner Relaxation in Ca-, Sm-and Nd-doped Ceria, Engineered Science, 2021, 15, 95-104, doi: 10.30919/es8d448.

[26] S. D. Satpute, J. S. Jagtap, P. K. Bhujbal, S. M. Sonar, P. K. Baviskar, S. R. Jadker, H. M. Pathan, Mercurochrome sensitized $\mathrm{ZnO} / \mathrm{In} 2 \mathrm{O} 3$ photoanode for dye-sensitized solar cell, ES Energy \& Environment, 2020, 9, 89-94, doi: 10.30919/esee8c720.

[27] P. Xie, Y. Li, Q. Hou, Tunneling-induced negative permittivity in $\mathrm{Ni} / \mathrm{MnO}$ nanocomposites by a bio-gel derived strategy, Journal of Materials Chemistry C, 2020, 8, 3029-3039, doi: 10.1039/C9TC06378A.

[28] J. Sun, X. Zhang, Q. Du, V. Murugadoss, D. Wu, Z. Guo, The contribution of conductive network conversion in thermal conductivity enhancement of polymer composite: a theoretical and experimental study, ES Materials \& Manufacturing, 2021, 13, 53-65, doi: 10.30919/esmm5f450.

[29] G. Qi, Y. Liu, L. Chen, P. Xie, D. Pan, Z. Shi, B. Quan, Y. Zhong, C. Liu, R. Fan, Z. Guo, Lightweight Fe3C@Fe/C nanocomposites derived from wasted cornstalks with highefficiency microwave absorption and ultrathin thickness, Advanced Composites and Hybrid Materials, 2021, 4, 1226-1238, doi: 10.1007/s42114-021-00368-0.

[30] S. Rehman, R. Ahmed, K. Ma, S. Xu, T. Tao, M. Aslam, M. Amir, J. Wang, Composite of strip-shaped ZIF-67 with polypyrrole: a conductive polymer-MOF electrode system for stable and high specific capacitance, Engineered Science, 2021, 
13, 71-78, doi: 10.30919/es8d1263.

[31] X. Yao, X. Kou, J. Qiu, Multi-walled carbon nanotubes/polyaniline composites with negative permittivity and negative permeability, Carbon, 2016, 107, 261-267, doi: 10.1016/j.carbon.2016.05.055.

[32] P. Xie, Y. Li, Q. Hou, Tunneling-induced negative permittivity in $\mathrm{Ni} / \mathrm{MnO}$ nanocomposites by a bio-gel derived strategy, Journal of Materials Chemistry C, 2020, 8, 3029-3039, doi: 10.1039/C9TC06378A.

[33] P. Xie, Y. Liu, M. Feng, Hierarchically porous $\mathrm{Co} / \mathrm{C}$ nanocomposites for ultralight high-performance microwave absorption, Advanced Composites and Hybrid, 2021, 4, 173-185, doi: 10.1007/s42114-020-00202-z.

[34] J. B. Pendry, A. J. Holden, D. J. Robbins, W. J. Stewart, Low frequency plasmons in thin-wire structures, Journal of Physics: Condensed Matter, 1998, 10, 4785-4809, doi: 10.1088/09538984/10/22/007.

[35] K. Yan, R. Fan, Z. Shi, Negative permittivity behavior and magnetic performance of perovskite La1- x Srx MnO3 at highfrequency, Journal of Materials Chemistry C, 2014, 2, 1028-1033, doi: 10.1039/C3TC31906G.

[36] S. Chui, L. Hu, Theoretical investigation on the possibility of preparing left-handed materials in metallic magnetic granular composites, Physical Review B, 2002, 65, 144407, doi: 10.1103/PhysRevB.65.144407.

[37] T. Tsutaoka, H. Massango, T. Kasagi, S. Yamamoto, K. Hatakeyama, Double negative electromagnetic properties of percolated Fe53Ni47/Cu granular composites, Applied Physics Letters, 2016, 108, 191904, doi: 10.1063/1.4949560.

[38] M. Han, X. Yin, H. Wu, Z. Hou, C. Song, C 2 MXenes with modified surface for high-performance electromagnetic absorption and shielding in the X-Band, ACS Applied Material Interfaces, 2016, 8, 21011-21019, doi: 10.1021/acsami.6b06455. [39] X. Zhang, X. Yan, Q. He, H. Wei, J. Long, Electrically conductive polypropylene nanocomposites with negative permittivity at low carbon nanotube loading levels, ACS Applied Materials \& Interfaces, 2015, 7, 6125-6138, doi: 10.1021/am5082183.

[40] B. Wen, M. Cao, M. Lu, Reduced graphene oxides: lightweight and high-efficiency electromagnetic interference shielding at elevated temperatures, Advanced Materials, 2014, 26, 3484-3489, doi: 10.1002/adma.201400108.

[41] M. Han, X. Yin, X. Li, Laminated and Two-Dimensional Carbon-Supported Microwave Absorbers Derived from MXenes, ACS Applied Materials \& Interfaces, 2017, 9, 20038-20045, doi: 10.1021/acsami.7b04602.

[42] K. Sun, P. Xie, Z. Wang, R. Fan, Flexible polydimethylsiloxane/multi-walled carbon nanotubes membranous metacomposites with negative permittivity, Polymer, 2017, 125, 50-57, doi: 10.1016/j.polymer.2017.07.083. [43] K. Sun, P. Yang, Q. He, R. Fan, Weakly negative permittivity of MWCNT/TiN/CCTO ternary ceramics sintered in argon and nitrogen atmosphere, Ceramics International, 2021, 47, $32297-$ 32302, doi: 10.1016/j.ceramint.2021.08.124.

[44] K. Sun, J. Qin, Z. Wang, R. Fan, Polyvinyl alcohol/carbon fibers composites with tunable negative permittivity behavior, Surfaces and Interfaces, 2020, 21, 100735, doi: 10.1016/j.surfin.2020.100735.

[45] F. Yao, W. Xie, M. Yang, Interfacial polymerized copolymers of aniline and phenylenediamine with tunable magnetoresistance and negative permittivity, Materials Today Physics, 2021, 21, 100502, doi: 10.1016/j.mtphys.2021.100502.

[46] Y. Xie, B. Zhou, A. Du, Slow-sound propagation in aerogelinspired hybrid structure with backbone and dangling branch, Advanced Composites and Hybrid Materials, 2021, 4, 248-256, doi: 10.1007/s42114-021-00234-z.

[47] X. Ji, Y. Zhong, C. Li, J. Chu, Nanoporous carbon aerogels for laser-printed wearable sensors, ACS Applied Nano Materials, 2021, 4, 6796-6804, doi: 10.1021/acsanm.1c00858.

[48] J. Guo, Z. Chen, Z. Guo, Tunable positive magnetoresistance of magnetic polyaniline nanocomposites, Advanced Composites and Hybrid Materials, 2021, 4, 534-542, doi: 10.1007/s42114021-00242-z.

[49] J. Guo, X. Li, H. Liu, Tunable magnetoresistance of coreshell structured polyaniline nanocomposites with 0-, 1-, and 2dimensional nanocarbons, Advanced Composites and Hybrid Materials, 2021, 4, 51-64, doi: 10.1007/s42114-021-00211-6.

[50] J. Guo, X. Li, Z. Chen, Magnetic NiFe2O4/polypyrrole nanocomposites with enhanced electromagnetic wave absorption Journal of Materials Science \& Technology, 2021, 108, 64-72, doi: 10.1016/j.jmst.2021.08.049.

\section{Author information}

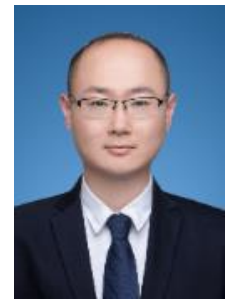

Peitao Xie, associate professor of materials science and engineering in Qingdao University. He mainly engaged in the research and development of novel metacomposites, negative dielectric metamaterials, and the structure design of electromagnetic interference shielding materials and microwave absorption materials.

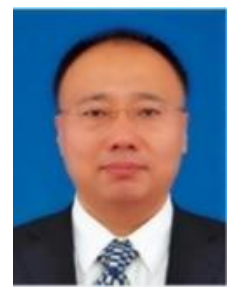

Chunzhao Liu, professor of materials science and engineering in Qingdao University. His main research field is biochemistry, studying the biochemical separation/catalytic applications by using functional magnetic beads modified with multi-functional groups, and realizing industrial applications in cell/protein magnetic separation and magnetic nanoenzyme catalysis.

Publisher's Note: Engineered Science Publisher remains neutral with regard to jurisdictional claims in published maps and institutional affiliations. 